\title{
SELETIVIDADE DE SULFENTRAZONE EM CULTIVARES DE SOJA E EFEI- TOS RESIDUAIS SOBRE CULTURAS SUCESSIVAS, EM SOLOS DE CERRADO
}

\author{
Francisco de Assis Rolim Pereira ${ }^{1}$, Sergio Luis A. Alvarenga ${ }^{2}$, \\ Sergio Otubo ${ }^{3}$, Ayrton Morceli $^{3}$ e Reinaldo Bazoni ${ }^{3}$
}

\author{
${ }^{1}$ UNIDERP. Rua Ceará, 333. Campo Grande, MS 79003-010 frolimp@terra.com.br \\ ${ }^{2} \mathrm{FMC}$ do Brasil Ind. e Com. S.A. Campo Grande, MS 79003-000 \\ ${ }^{3}$ EMPAER-MS. Caixa Postal 472. Campo Grande, MS 79003-902
}

\begin{abstract}
RESUMO
As características edáficas, em interação com o clima e a cultivar semeada, podem causar fitotoxicidade na cultura em área tratada com herbicida residual. Com o objetivo de avaliar a seletividade do herbicida sulfentrazone sobre 21 cultivares de soja e verificar os efeitos residuais sobre culturas sucessivas à soja, foram conduzidos dois ensaios em solos de Cerrado, município de Campo Grande, MS. O ensaio 1 foi instalado em solo de origem de Areia Quartzosa, contendo $8 \%$ de argila, $2 \%$ de silte, $90 \%$ de areia total e $2,3 \%$ de matéria orgânica. O ensaio 2 foi instalado em Latossolo vermelho-escuro, contendo $35 \%$ de argila, $11 \%$ de silte e $54 \%$ de areia total. Os tratamentos nos dois ensaios foram: sulfentrazone nas doses de 500 e $1000 \mathrm{~g} / \mathrm{ha}$, diclosulam nas doses de $35 \mathrm{e} 70 \mathrm{~g} / \mathrm{ha}$, sulfentrazone+diclosulam a $250+25$ e $500+50 \mathrm{~g} / \mathrm{ha}$ e testemunha capinada. Os resultados mostraram que a fitotoxicidade mais expressiva foi detectada apenas no ensaio $\mathrm{n}^{\circ} 1$ (solo arenoso), através de sulfentrazone a $1000 \mathrm{~g} / \mathrm{ha}$ e diclosulam a $70 \mathrm{~g} / \mathrm{ha}$, sobre a cultivar Conquista e com menor intensidade sobre as cultivares Taquari e Paiaguás. As culturas de milheto, aveia e sorgo, plantadas em sucessão à soja, apresentaram sintomas evidentes de fitotoxicidade através dos tratamentos com sulfentrazone a $1000 \mathrm{~g} / \mathrm{ha} \mathrm{e}$ diclosulam a $70 \mathrm{~g} / \mathrm{ha}$ Os tratamentos químicos, nos dois ensaios, apresentaram controle eficiente das plantas daninhas Digitaria horizontalis, Amaranthus viridis, Ipomoea grandifolia, Portulaca oleraceae e Bidens pilosa. Conclui-se que os herbicidas sulfentrazone e diclosulam, aplicados em pré-emergência, podem apresentar danos a algumas cultivares de soja quando plantadas em solos com textura arenosa.
\end{abstract}

Palavras-chave: herbicidas, textura de solo, plantas daninhas.

\section{ABSTRACT \\ Selectivity of sulfentrazone on soybean cultivars and the residual effects on rotation crops, in Savanna soils}

Edaphic characteristics, in interaction with the climate and the specific cultivar, may cause crop toxicity in an area treated with a residual herbicide. Two experiments were carried out on two Cerrado soils, in Campo Grande (MS), to evaluate 
Francisco de Assis Rolim Pereira et al.

the selectivity of sulfentrazone on 21 soybean cultivars, as well as the residual effects on successive soybean crops. The first experiment was on a sandy soil, with $8 \%$ clay, $2 \%$ lime, $90 \%$ total sand and $2.3 \%$ organic matter. The second experiment was carried out on a soil with $35 \%$ clay, $11 \%$ lime and $54 \%$ sand. Treatments in both cases were: sulfentrazone at 500 and 1000 $\mathrm{g} / \mathrm{ha}$, diclosulam at 35 and $70 \mathrm{~g} / \mathrm{ha}$, sulfentrazone+diclosulam at $250+25$ and $500+50 \mathrm{~g} / \mathrm{ha}$, and a control where the weeds were mechanically cut. Results showed that higher phytotoxicity was found only in the experiment on sandy soil, with sulfentrazone at $1000 \mathrm{~g} / \mathrm{ha}$ and diclosulam at $70 \mathrm{~g} / \mathrm{ha}$, in Conquista cultivar, and at a lower level in the Taquari and Paiguás cultivar. Millet, oat and sorghum sown in sucession to soybean, showed phytotoxicity symptoms to sulfentrazone (1000 g/ha) and diclosulam (70 g/ha). Chemical treatments, in both experiments, showed efficient control of Digitaria horizontalis, Amaranthus viridis, Ipomoea grandifolia, Portulaca oleraceae and Bidens pilosa. It was concluded that preemergence aplications of diclosulam can injure some soybean cultivars on sandy soil.

Key words: herbicides, soil texture, weeds.

\section{INTRODUÇÃO}

Durante o período de desenvolvimento e posteriormente a nível de lavoura, comprovou-se que o herbicida sulfentrazone apresenta excelente eficiência no controle de plantas daninhas na cultura da soja, com largo espectro de ação. No tocante à seletividade do produto à cultura da soja, alguns resultados experimentais geraram informações detectando danos fitotóxicos, ocorridos em casos isolados. Dentre as hipóteses levantadas, destacam-se a influência do tipo de solo, principalmente quanto aos teores de argila e de matéria orgânica e a interação do ambiente com a cultivar plantada. Walker et al. (1992), relatam que cultivares de soja variaram em sua tolerância ao produto, aplicado em pré-emergência, em solos com baixo teor de argila. Conforme Dayan et al. (1997), sulfentrazone é classificado, pelo seu mecanismo de ação, como inibidor de PROTOX. Estes herbicidas causam acúmulo de protoporfirina IX, que na presença de luz e de oxigênio molecular gera oxigênio elementar, causando destruição da membrana celular, provocando rápida dessecação e necrose dos tecidos. De acordo com Duke et al (1996), não há registro de casos de espécies resistentes aos herbicidas com este mecanismo de ação. São várias as espécies de plantas daninhas suscetíveis à ação de sulfentrazone, sendo registradas 28 espécies, abrangendo plantas de folhas largas (latifoliadas) e de folhas estreitas principalmente gramíneas (Rodrigues \& Almeida, 1998).

Dada a importância deste herbicida nos programas de rotação de produtos e de manejo de plantas daninhas na cultura da soja e em culturas sucessivas, faz-se necessário a realização de pesquisas visando detectar possível suscetibilidade de cultivares de soja, quando semeadas em ambiente desfavorável, e os efeitos residuais de alguns herbicidas sobre culturas semeadas no outono/inverno. Os principais objetivos deste trabalho foram: avaliar a seletividade do herbicida sulfentrazone sobre 21 cultivares de soja em solos de textura arenosa e de textura média, num ambiente de Cerrado e verificar a ação residual de sulfentrazone sobre algumas culturas semeadas em sucessão à soja.

\section{MATERIAL E MÉTODOS}

O ensaio 1 foi instalado em 27/11/1999, no Campus III da Uniderp, localizado no município de Campo Grande,MS. O ensaio 2 foi instalado em 13/12/1999, na Estação Experimental da EMPAER-MS, localizada no município de Campo Grande, MS. As culturas em sucessão foram semeadas em 28/04/2000 (ensaio 1) e em 26/05/2000 (ensaio 2). Nos dois ensaios foram semeadas as cultivares Bacuri, Pioneira, Uirapuru, Taquari, Curió, Campo Grande, Xingu, Tucano, Parecis, Cristalina RC, Garça Branca, CAC1, Piapara, Canário, Conquista, Paiaguás, Surubi, Pintado, Suprema, FT-2000 e FT-Estrela. A lista de cultivares foi composta com base nos materiais genéticos recomendados para a região de Cerrados, conforme EMBRAPA (1999). O espaçamento utilizado foi de $50 \mathrm{~cm}$ entre linhas com $25 \mathrm{se}-$ mentes por metro linear, proporcionando uma densidade média de 40 plantas $/ \mathrm{m}^{2}$. Após a colheita da soja semeou-se na área experimental dos dois ensaios, em faixas, culturas em sucessão à soja visando avaliar a ação residual dos produtos no seu desenvolvimento. As espécies semeadas foram: milheto (Pennisetum americanum), aveia-preta (Avena sativa), sorgo (Sorghum bicolor, cv. Z-822), milho (Zea mays , cv. Z-8202), feijão (Phaseolus vulgaris, cv. carioca) e algodão (Gossypium hirsutum, cv. Ita 90). As áreas dos ensaios foram adubadas com $350 \mathrm{~kg} / \mathrm{ha}$ da fórmula $05-25$ 20. Os tratos culturais empregados seguiram as recomendações para a cultura (EMBRAPA, 1999). Para as culturas sucessivas não houve adubação. As características químicas e físicas dos solos são apresentadas na Tabela 1. No ensaio 10 solo é do tipo Areia Quartzosa, distrófica, relevo suaveondulado, fase sob Cerrado e textura arenosa. No ensaio 2 o solo é um Latossolo vermelho-escuro, distrófico, relevo suave-ondulado, fase sob Cerrado e textura argilo-arenosa (textura média).

A distribuição da comunidade infestantes nos dois ensaios está apresentada na Tabela 2 .

Os ensaios foram delineados em blocos ao acaso e parcelas subdivididas, sendo as parcelas os tratamentos químicos e as subparcelas as cultivares, com 3 repetições e 147 
Tabela 1. Resultados das analíses quimica (profundidade de 0-20 cm) e física do solo nos ensaios 1 e 2. Campo Grande, MS. 1999/2000.

\begin{tabular}{lrc}
\hline & Ensaio 1 & Ensaio 2 \\
\hline Análise química & 6,5 & 6,0 \\
$\mathrm{pH}\left(\mathrm{H}_{2} \mathrm{O}\right)$ & 8,0 & 5,0 \\
$\mathrm{P}(\mathrm{ppm})^{1}$ & 25,0 & 63,0 \\
$\mathrm{~K}(\mathrm{ppm})$ & 2,3 & 2,6 \\
M.O. (\%) & 0,0 & 0,1 \\
A.1 (m.e.) & 2,9 & 4,9 \\
Ca+Mg (m.o.) & & \\
Análise física & 90 & 54 \\
Areia (\%) & 2 & 11 \\
Silte (\%) & 8 & 35 \\
Argila (\%) & & \\
\hline
\end{tabular}

${ }^{1}$ Extraído pelo método Mehlich I.

tratamentos. Os tratamentos químicos foram aplicados em sentido transversal às linhas de semeadura das cultivares. As culturas em sucessão foram semeadas em faixas na área experimental, abrangendo todas as parcelas (tratamentos químicos) do ensaio. Para aplicação dos herbicidas utilizou-se um pulverizador costal de pressão constante, pressurizado por $\mathrm{CO}_{2}$, munido de uma barra com 4 bicos tipo leque 110.02 e espaçados em 0,5 m. O volume de calda aplicado foi de 200 litros/ha. Durante as aplicações a temperatura média ambiente foi de $26^{\circ} \mathrm{C}$, umidade relativa do ar $48 \%$ e ventos moderados a praticamente ausentes no ensaio 1 e temperatura média ambiente de $28^{\circ} \mathrm{C}$, umidade relativa do ar $52 \%$ e ventos moderados a praticamente ausentes, no ensaio 2 . Realizaram-se avaliações de fitotoxicidade e de eficiência aos 30, 45 e 60 dias após aplicação dos tratamentos (DAT). Empregou-se o método de avaliação visual, através de uma escala de 0 a 100 , onde: $0=$ nenhuma injúria na planta e 100 $=$ morte total da planta. Para eficiência considerou-se a nota 80 como o padrão mínimo aceitável; para fitotoxicidade, con-

Tabela 2. Densidade média de plantas daninhas nas parcelas testemunhas, aos $\mathbf{3 0}$ dias após a aplicação dos tratamentos. Campo Grande, MS. $1999 / 2000$.

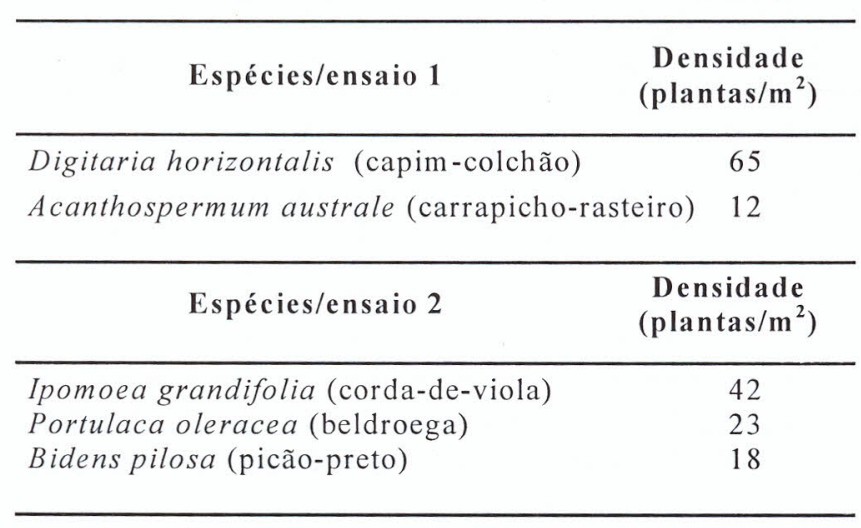

siderou-se 40 a nota máxima aceitável, passível de recuperação da cultura sem perspectivas de redução no rendimento econômico. Sobre as culturas em sucessão realizaram-se avaliações de fitotoxicidade aos 30, 45 e 60 dias após a semeadura (DAS), empregando-se o mesmo método de avaliação visual citado anteriormente. Para análise dos resultados de eficiência, procedeu-se análise de variância e as médias comparadas pelo teste de Tukey ao nível de 5\% de probabilidade.

\section{RESULTADOS E DISCUSSÃO}

Os resultados mostraram que entre os dois ambientes estudados, o solo de textura mista (ensaio 2), apresenta maior segurança ao uso do herbicida sulfentrazone e que em solos com textura arenosa deve ser evitado o uso deste produto. Ressalta-se que os tratamentos que apresentaram maior índice de fitotoxicidade na soja foram aqueles com o dobro da dose recomendada. Todavia, por prudência e segurança este herbicida não deve ser indicado para solos leves, principalmente quando se utilizam cultivares mais suscetíveis. Embora com menor intensidade, as mesmas considerações são creditadas ao herbicida diclosulam.

No ensaio 1, realizado em solo arenoso, os resultados das avaliações de fitotoxicidade aos 30 DAT (Tabela 3), mostraram que os tratamentos com sulfentrazone+diclosulam $(250+25 \mathrm{~g} / \mathrm{ha})$ e diclosulam (35 g/ha), não apresentaram nenhum sintoma fitotóxico sobre as cultivares. O tratamento com sulfentrazone a $500 \mathrm{~g} /$ ha apresentou avaliação média de $10 \%$ de fitotoxicidade sobre as cultivares Conquista e Taquari, $4 \%$ sobre a cv. Paiaguás e $0 \%$ sobre as demais cultivares. Sulfentrazone a $1000 \mathrm{~g} /$ ha apresentou fitotoxidade média de $35 \%$ sobre a cv. Conquista, $15 \%$ sobre a Taquari, $11 \%$ sobre a Paiaguás, $10 \%$ sobre as cultivares Pintado, Suprema e FTEstrela, $8 \%$ sobre a cv. Bacuri, 7\% sobre a Tucano, $6 \%$ sobre a Surubi e $0 \%$ sobre as demais. O tratamento com sulfentrazone+diclosulam $(500+50 \mathrm{~g} / \mathrm{ha})$, apresentou média de $15 \%$ de fitotoxicidade sobre a cv. Conquista, $10 \%$ sobre a cv. Paiaguás e $0 \%$ sobre as demais. Diclosulam a $70 \mathrm{~g} / \mathrm{ha}$ apresentou média de $15 \%$ de injúria sobre a cv. Conquista e $0 \%$ sobre as demais cultivares. Nas avaliações aos 45 DAT (Tabela 4), verificou-se sintomas de fitotoxicidade através de sulfentrazone a $500 \mathrm{~g} /$ ha com média de $10 \%$ sobre a cv. Conquista, $8 \%$ sobre a cv. Taquari e $2 \%$ sobre a cv. Paiaguás. $\mathrm{O}$ tratamento com sulfentrazone a $1000 \mathrm{~g} / \mathrm{ha}$, apresentou fitotoxicidade média de $38 \%$ sobre a cv. Conquista, $17 \%$ sobre a Surubi, $15 \%$ sobre a cv. Taquari, $13 \%$ sobre as cultivares Bacuri, Suprema e FT-Estrela, $12 \%$ sobre a cv. Pintado, $11 \%$ sobre a Paiaguás e $3 \%$ sobre a FT-2000. Sulfentrazone+diclosulam $(500+50 \mathrm{~g} / \mathrm{ha})$ apresentou fitotoxicidade média de $15 \%$ sobre a cv. Conquista e $11 \%$ sobre a Paiaguás. Diclosulam a $70 \mathrm{~g} / \mathrm{ha}$ apresentou fitotoxicidade média de $13 \%$ sobre a cv. Conquista e $11 \%$ sobre a Taquari. Aos 60 DAT (Tabela 5), foram constatados sintomas de fitotoxicidade apenas através dos tratamentos 
com sulfentrazone a $1000 \mathrm{~g} / \mathrm{ha}$, sobre as cultivares Conquista $(25 \%)$, Paiaguás $(10 \%)$ e Taquari $(10 \%)$.

No ensaio 2, solo misto, a fitotoxicidade ocorreu com menor intensidade que no ensaio 1 , evidenciando a influência do teor de argila sobre a ação seletiva dos produtos avaliados. Nas avaliações aos 30 DAT (Tabela 6) os sintomas fitotóxicos mais expressivos foram detectados através do tratamento com sulfentrazone a $1000 \mathrm{~g} /$ ha , que apresentou média de $10 \%$ de fitotoxicidade sobre a cv. Conquista. Aos 45 DAT os resultados foram semelhantes aos obtidos na avaliação anterior e, aos 60 DAT, nenhum tratamento apresentava sintomas aparentes de fitotoxicidade.

Tabela 3 - Avaliações de fitotoxicidade aos 30 DAT (solo arenoso). Campo Grande, MS. 1999/2000

\begin{tabular}{|c|c|c|c|c|c|c|c|c|c|c|c|c|c|c|c|c|c|c|c|c|c|}
\hline \multirow[t]{2}{*}{ PRODUTO } & \multirow{2}{*}{$\begin{array}{l}\text { DOSE } \\
(\mathrm{g} / \mathrm{ha})\end{array}$} & \multicolumn{20}{|c|}{ FITOTOXICIDADE (\%) / CULTIVAR DE SOJA } \\
\hline & & $\mathbf{A}$ & B & $\mathrm{C}$ & $\mathbf{D}$ & $\mathbf{E}$ & $\mathbf{F}$ & $\mathrm{G}$ & $\mathbf{H}$ & I & $\mathbf{J}$ & $\mathbf{L}$ & $\mathbf{M}$ & $\mathbf{N}$ & O & $\mathbf{P}$ & $\mathbf{Q}$ & $\mathbf{R}$ & $\mathbf{S}$ & $\mathrm{T}$ & $\mathbf{U}$ \\
\hline Testemunha & -- & 0 & 0 & 0 & 0 & 0 & 0 & 0 & 0 & 0 & 0 & 0 & 0 & 0 & 0 & 0 & 0 & 0 & 0 & 0 & 0 \\
\hline Sulfentrazone & 500 & 0 & 0 & 0 & 10 & 0 & 0 & 0 & 0 & 0 & 0 & 0 & 0 & 0 & 10 & 4 & 0 & 0 & 0 & 0 & 0 \\
\hline Sulfentrazone & 1000 & 8 & 0 & 0 & 15 & 0 & 0 & 0 & 5 & 0 & 0 & 0 & 0 & 0 & 35 & 10 & 0 & 10 & 10 & 0 & 10 \\
\hline Sulfentrazone + diclosulam & $250+25$ & 0 & 0 & 0 & 0 & 0 & 0 & 0 & 0 & 0 & 0 & 0 & 0 & 0 & 0 & 0 & 0 & 0 & 0 & 0 & 0 \\
\hline Sulfentrazone + diclosulam & $500+50$ & 0 & 0 & 0 & 0 & 0 & 0 & 0 & 0 & 0 & 0 & 0 & 0 & 0 & 15 & 10 & 0 & 0 & 0 & 0 & 0 \\
\hline Diclosulam & 35 & 0 & 0 & 0 & 0 & 0 & 0 & 0 & 0 & 0 & 0 & 0 & 0 & 0 & 0 & 0 & 0 & 0 & 0 & 0 & 0 \\
\hline Diclosulam & 70 & 0 & 0 & 0 & 10 & 0 & 0 & 0 & 0 & 0 & 0 & 0 & 0 & 0 & 15 & 0 & 0 & 0 & 0 & 0 & 0 \\
\hline
\end{tabular}

$\mathrm{A}=$ Bacuri, $\mathrm{B}=$ Pioneira, $\mathrm{C}=$ Uirapuru, $\mathrm{D}=$ Taquari, $\mathrm{E}=$ Curió, $\mathrm{F}=$ Campo Grande, $\mathrm{G}=\mathrm{Xingu}, \mathrm{H}=$ Tucano, $\mathrm{I}=$ Parecis, $\mathrm{J}=\mathrm{Cristalina}$ $\mathrm{RC}, \mathrm{K}=$ Garça Branca, $\mathrm{L}=\mathrm{CAC}-1, \mathrm{M}=$ Piapara, $\mathrm{N}=$ Canário, $\mathrm{O}=$ Conquista, $\mathrm{P}=$ Paiaguás, $\mathrm{Q}=$ Surubi, $\mathrm{R}=\mathrm{Pintado}, \mathrm{S}=$ Suprema, $\mathrm{T}=\mathrm{FT}-2000, \mathrm{U}=\mathrm{FT}$-Estrela

Tabela 4 - Avaliações de fitotoxicidade aos 45 DAT (solo arenoso). Campo Grande, MS. 1999/2000.

\begin{tabular}{|c|c|c|c|c|c|c|c|c|c|c|c|c|c|c|c|c|c|c|c|c|c|}
\hline \multirow[t]{2}{*}{ PRODUTO } & \multirow{2}{*}{$\begin{array}{r}\text { DOSE } \\
(\mathrm{g} / \mathrm{ha})\end{array}$} & \multicolumn{20}{|c|}{ FITOTOXICIDADE (\%) / CULTIVAR DE SOJA } \\
\hline & & A & B & $\mathrm{C}$ & $\mathbf{D}$ & $\mathbf{E}$ & $\mathbf{F}$ & G & H & I & $\mathbf{J}$ & $\mathbf{L}$ & M & $\mathbf{N}$ & $\mathbf{O}$ & $\mathbf{P}$ & $\mathbf{Q}$ & $\mathbf{R}$ & $\mathbf{S}$ & $\mathbf{T}$ & $\mathbf{U}$ \\
\hline Testemunha & -- & 0 & 0 & 0 & 0 & 0 & 0 & 0 & 0 & 0 & 0 & 0 & 0 & 0 & 0 & 0 & 0 & 0 & 0 & 0 & 0 \\
\hline Sulfentrazone & 500 & 0 & 0 & 0 & 8 & 0 & 0 & 0 & 0 & 0 & 0 & 0 & 0 & 0 & 10 & 2 & 0 & 0 & 0 & 0 & 0 \\
\hline Sulfentrazone & 1000 & 13 & 0 & 0 & 15 & 0 & 0 & 0 & 8 & 0 & 0 & 0 & 0 & 0 & 38 & 11 & 17 & 12 & 13 & 3 & 13 \\
\hline Sulfentrazone+diclosulam & $250+25$ & 0 & 0 & 0 & 0 & 0 & 0 & 0 & 0 & 0 & 0 & 0 & 0 & 0 & 0 & 0 & 0 & 0 & 0 & 0 & 0 \\
\hline Sulfentrazone+diclosulam & $500+50$ & 0 & 0 & 0 & 0 & 0 & 0 & 0 & 0 & 0 & 0 & 0 & 0 & 0 & 15 & 11 & 0 & 0 & 0 & 0 & 0 \\
\hline Diclosulam & 35 & 0 & 0 & 0 & 0 & 0 & 0 & 0 & 0 & 0 & 0 & 0 & 0 & 0 & 0 & 0 & 0 & 0 & 0 & 0 & 0 \\
\hline Diclosulam & 70 & 0 & 0 & 0 & 11 & 0 & 0 & 0 & 0 & 0 & 0 & 0 & 0 & 0 & 13 & 0 & 0 & 0 & 0 & 0 & 0 \\
\hline
\end{tabular}

$\mathrm{A}=$ Bacuri, $\mathrm{B}=$ Pioneira, $\mathrm{C}=$ Uirapuru, $\mathrm{D}=$ Taquari, $\mathrm{E}=$ Curió, $\mathrm{F}=$ Campo Grande, $\mathrm{G}=\mathrm{Xingu}, \mathrm{H}=$ Tucano, $\mathrm{I}=$ Parecis, $\mathrm{J}=\mathrm{Cristalina}$ $\mathrm{RC}, \mathrm{K}=$ Garça Branca, $\mathrm{L}=\mathrm{CAC}-1, \mathrm{M}=$ Piapara, $\mathrm{N}=$ Canário, $\mathrm{O}=$ Conquista, $\mathrm{P}=$ Paiaguás, $\mathrm{Q}=$ Surubi, $\mathrm{R}=\mathrm{Pintado}, \mathrm{S}=\mathrm{Suprema}$, $\mathrm{T}=\mathrm{FT}-2000, \mathrm{U}=\mathrm{FT}-$ Estrela

Tabela 5 - Avaliações de fitotoxicidade aos 60 DAT (solo arenoso). Campo Grande, MS. 1999/2000.

\begin{tabular}{|c|c|c|c|c|c|c|c|c|c|c|c|c|c|c|c|c|c|c|c|c|c|}
\hline \multirow[t]{2}{*}{ PRODUTO } & \multirow{2}{*}{$\begin{array}{r}\text { DOSE } \\
\text { (g/ha) }\end{array}$} & \multicolumn{20}{|c|}{ FITOTOXICIDADE (\%) / CULTIVAR DE SOJA } \\
\hline & & $\mathbf{A}$ & B & $\mathbf{C}$ & D & $\mathbf{E}$ & $\mathbf{F}$ & G & $\mathbf{H}$ & I & $\mathbf{J}$ & $\mathbf{L}$ & $\mathbf{M}$ & $\mathbf{N}$ & $\mathbf{O}$ & $\mathbf{P}$ & Q & $\mathbf{R}$ & $\mathbf{S}$ & $\mathbf{T}$ & $\mathbf{U}$ \\
\hline Testemunha & -- & 0 & 0 & 0 & 0 & 0 & 0 & 0 & 0 & 0 & 0 & 0 & 0 & 0 & 0 & 0 & 0 & 0 & 0 & 0 & 0 \\
\hline Sulfentrazone & 500 & 0 & 0 & 0 & 0 & 0 & 0 & 0 & 0 & 0 & 0 & 0 & 0 & 0 & 0 & 2 & 0 & 0 & 0 & 0 & 0 \\
\hline Sulfentrazone & 1000 & 0 & 0 & 0 & 10 & 0 & 0 & 0 & 0 & 0 & 0 & 0 & 0 & 0 & 25 & 10 & 0 & 0 & 0 & 0 & 0 \\
\hline Sulfentrazone+diclosulam & $250+25$ & 0 & 0 & 0 & 0 & 0 & 0 & 0 & 0 & 0 & 0 & 0 & 0 & 0 & 0 & 0 & 0 & 0 & 0 & 0 & 0 \\
\hline Sulfentrazone+diclosulam & $500+50$ & 0 & 0 & 0 & 0 & 0 & 0 & 0 & 0 & 0 & 0 & 0 & 0 & 0 & 0 & 0 & 0 & 0 & 0 & 0 & 0 \\
\hline Diclosulam & 35 & 0 & 0 & 0 & 0 & 0 & 0 & 0 & 0 & 0 & 0 & 0 & 0 & 0 & 0 & 0 & 0 & 0 & 0 & 0 & 0 \\
\hline Diclosulam & 70 & 0 & 0 & 0 & 0 & 0 & 0 & 0 & 0 & 0 & 0 & 0 & 0 & 0 & 0 & 0 & 0 & 0 & 0 & 0 & 0 \\
\hline
\end{tabular}

$\mathrm{A}=$ Bacuri, $\mathrm{B}=$ Pioneira, $\mathrm{C}=$ Uirapuru, $\mathrm{D}=$ Taquari, $\mathrm{E}=$ Curió, $\mathrm{F}=$ Campo Grande, $\mathrm{G}=\mathrm{X}$ ingu, $\mathrm{H}=$ Tucano, $\mathrm{I}=$ Parecis, $\mathrm{J}=\mathrm{Cristalina}$ $\mathrm{RC}, \mathrm{K}=$ Garça Branca, $\mathrm{L}=\mathrm{CAC}-1, \mathrm{M}=$ Piapara, $\mathrm{N}=$ Canário, $\mathrm{O}=$ Conquista, $\mathrm{P}=$ Paiaguás, $\mathrm{Q}=$ Surubi, $\mathrm{R}=\mathrm{Pintado}, \mathrm{S}=$ Suprema, $\mathrm{T}=\mathrm{FT}-2000, \mathrm{U}=\mathrm{FT}-$ Estrela 
Os sintomas de fitotoxicidade caracterizavam-se sempre por necroses foliares e/ou redução do porte das plantas.

Os resultados das avaliações de eficiência (Tabelas 7 e 8) registraram que os tratamentos químicos foram eficazes no controle das plantas daninhas avaliadas.

Os efeitos residuais dos tratamentos químicos apresentaram sintomas fitotóxicos apenas na área experimental do ensaio 1 (solo arenoso), através dos tratamentos com sulfentrazone a $1000 \mathrm{~g} / \mathrm{ha}$ e diclosulam a $70 \mathrm{~g} / \mathrm{ha}$, sobre as culturas sucessivas de milheto, aveia, sorgo e com menor intensidade sobre a cultura do algodão (Tabela 9). No solo de textura argilo-arenosa (ensaio 2), não foram constatados sintomas significativos de fitotoxicidade, conferindo segurança ao plantio das culturas em sucessão à soja.

Tabela 6 - Avaliações de fitotoxicidade aos 30 DAT (solo argilo-arenoso). Campo Grande, MS. 1999/2000.

\begin{tabular}{|c|c|c|c|c|c|c|c|c|c|c|c|c|c|c|c|c|c|c|c|c|c|}
\hline \multirow[t]{2}{*}{ PRODUTO } & \multirow{2}{*}{$\begin{array}{c}\text { DOSE } \\
\text { (g/ha) }\end{array}$} & \multicolumn{20}{|c|}{ FITOTOXICIDADE (\%) / CULTIVAR DE SOJA } \\
\hline & & $\mathbf{A}$ & B & $\mathrm{C}$ & D & $\mathbf{E}$ & $\mathbf{F}$ & $\mathbf{G}$ & $\mathbf{H}$ & I & $\mathbf{J}$ & $\mathbf{L}$ & M & $\mathbf{N}$ & $\mathbf{O}$ & $\mathbf{P}$ & $\mathbf{Q}$ & $\mathbf{R}$ & $\mathbf{S}$ & $\mathbf{T}$ & $\mathbf{U}$ \\
\hline Testemunha & - & 0 & 0 & 0 & 0 & 0 & 0 & 0 & 0 & 0 & 0 & 0 & 0 & 0 & 0 & 0 & 0 & 0 & 0 & 0 & 0 \\
\hline Sulfentrazone & 500 & 0 & 0 & 0 & 0 & 0 & 0 & 0 & 0 & 0 & 0 & 0 & 0 & 0 & 0 & 0 & 0 & 0 & 0 & 0 & 0 \\
\hline Sulfentrazone & 1000 & 0 & 0 & 0 & 0 & 0 & 0 & 0 & 0 & 0 & 0 & 0 & 0 & 0 & 10 & 0 & 0 & 0 & 0 & 0 & 0 \\
\hline Sulfentrazone +diclosulam & $250+25$ & 0 & 0 & 0 & 0 & 0 & 0 & 0 & 0 & 0 & 0 & 0 & 0 & 0 & 0 & 0 & 0 & 0 & 0 & 0 & 0 \\
\hline Sulfentrazone+diclosulam & $500+50$ & 0 & 0 & 0 & 0 & 0 & 0 & 0 & 0 & 0 & 0 & 0 & 0 & 0 & 5 & 0 & 0 & 0 & 0 & 0 & 0 \\
\hline Diclosulam & 35 & 0 & 0 & 0 & 0 & 0 & 0 & 0 & 0 & 0 & 0 & 0 & 0 & 0 & 0 & 0 & 0 & 0 & 0 & 0 & 0 \\
\hline Diclosulam & 70 & 0 & 0 & 0 & 5 & 0 & 0 & 0 & 0 & 0 & 0 & 0 & 0 & 0 & 5 & 0 & 0 & 0 & 0 & 0 & 0 \\
\hline
\end{tabular}

$\mathrm{A}=$ Bacuri, $\mathrm{B}=$ Pioneira, $\mathrm{C}=$ Uirapuru, $\mathrm{D}=$ Taquari, $\mathrm{E}=$ Curió, $\mathrm{F}=$ Campo Grande, $\mathrm{G}=$ Xingu, $\mathrm{H}=$ Tucano, $\mathrm{I}=$ Parecis, $\mathrm{J}=$ Cristalina $\mathrm{RC}, \mathrm{K}=$ Garça Branca, $\mathrm{L}=\mathrm{CAC}-1, \mathrm{M}=$ Piapara, $\mathrm{N}=$ Canário, $\mathrm{O}=$ Conquista, $\mathrm{P}=$ Paiaguás, $\mathrm{Q}=$ Surubi, $\mathrm{R}=\mathrm{Pintado}, \mathrm{S}=$ Suprema, $\mathrm{T}=\mathrm{FT}-2000, \mathrm{U}=\mathrm{FT}-\mathrm{Estrela}$

Tabela 7 - Avaliações de eficiência de controle. Ensaio 1, Solo arenoso. Campo Grande, MS. 1999/2000.

\begin{tabular}{|c|c|c|c|c|c|c|c|}
\hline \multirow{3}{*}{ PRODUTOS } & \multirow{3}{*}{$\begin{array}{l}\text { DOSE } \\
\text { (g/ha) }\end{array}$} & \multicolumn{6}{|c|}{ CONTROLE (\%) } \\
\hline & & \multicolumn{3}{|c|}{ Digitaria horizontalis } & \multicolumn{3}{|c|}{ Amaranthus viridis } \\
\hline & & 30 DAT & 45 DAT & $60 \mathrm{DAT}$ & $30 \mathrm{DAT}$ & 45 DAT & $60 \mathrm{DAT}$ \\
\hline Testemunha & - & $0 \mathrm{c}$ & $0 \mathrm{c}$ & $0 \mathrm{c}$ & $0 \mathrm{c}$ & $0 \mathrm{c}$ & $0 \mathrm{c}$ \\
\hline Sulfentrazone & 500 & $95 \mathrm{a}$ & $90 \mathrm{ab}$ & $85 \mathrm{~b}$ & 95 a & $90 \mathrm{~b}$ & $90 \mathrm{~b}$ \\
\hline Sulfentrazone & 1000 & $100 \mathrm{a}$ & $100 \mathrm{a}$ & $95 \mathrm{a}$ & $100 \mathrm{a}$ & $100 \mathrm{a}$ & $95 \mathrm{ab}$ \\
\hline Sulfentrazone + diclosulam & $250+25$ & $90 \mathrm{ab}$ & $90 \mathrm{ab}$ & $85 \mathrm{~b}$ & $90 \mathrm{~b}$ & $90 \mathrm{~b}$ & $90 \mathrm{~b}$ \\
\hline Sulfentrazone+diclosulam & $500+50$ & $100 \mathrm{ab}$ & $100 \mathrm{a}$ & 95 a & $100 \mathrm{a}$ & $100 \mathrm{a}$ & $100 \mathrm{a}$ \\
\hline Diclosulam & 35 & $80 \mathrm{~b}$ & $85 \mathrm{~b}$ & $85 \mathrm{~b}$ & $90 \mathrm{~b}$ & $90 \mathrm{~b}$ & $90 \mathrm{~b}$ \\
\hline Diclosulam & 70 & $90 \mathrm{ab}$ & $90 \mathrm{ab}$ & $90 \mathrm{ab}$ & $100 \mathrm{a}$ & $100 \mathrm{a}$ & $95 \mathrm{ab}$ \\
\hline C.V. (\%) & - & 4,2 & 6,1 & 4,0 & 4,1 & 5,1 & 4,7 \\
\hline
\end{tabular}

Tabela 8 - Avaliações de eficiência de controle . Ensaio 2, Solo argilo-arenoso. Campo Grande, MS. $1999 / 2000$.

\begin{tabular}{|c|c|c|c|c|c|c|c|c|c|c|}
\hline \multirow{3}{*}{ PRODUTOS } & \multirow{3}{*}{$\begin{array}{c}\text { DOSE } \\
\text { (g/ha) }\end{array}$} & \multicolumn{9}{|c|}{ CONTROLE (\%) } \\
\hline & & \multicolumn{3}{|c|}{ Ipomoea grandifolia } & \multicolumn{3}{|c|}{ Portulaca oleraceae } & \multicolumn{3}{|c|}{ Bidens pilosa } \\
\hline & & $30 \mathrm{DAT}$ & $45 \mathrm{DAT}$ & $60 \mathrm{DAT}$ & $30 \mathrm{DAT}$ & $45 \mathrm{DAT}$ & $60 \mathrm{DAT}$ & $30 \mathrm{DAT}$ & $45 \mathrm{DAT}$ & $60 \mathrm{DAT}$ \\
\hline Testemunha & -- & $0 \mathrm{c}$ & $0 \mathrm{~b}$ & $0 \mathrm{~b}$ & $0 \mathrm{~b}$ & $0 \mathrm{c}$ & $0 \mathrm{c}$ & $0 \mathrm{~b}$ & $0 \mathrm{c}$ & $0 \mathrm{~b}$ \\
\hline Sulfentrazone & 500 & $95 \mathrm{a}$ & $90 \mathrm{a}$ & $85 \mathrm{a}$ & $100 \mathrm{a}$ & $90 \mathrm{ab}$ & $90 \mathrm{ab}$ & $90 \mathrm{a}$ & $90 \mathrm{ab}$ & $90 \mathrm{a}$ \\
\hline Sulfentrazone & 1000 & $100 \mathrm{a}$ & $90 \mathrm{a}$ & $90 \mathrm{a}$ & $100 \mathrm{a}$ & 95 a & $95 \mathrm{a}$ & $100 \mathrm{a}$ & $100 \mathrm{a}$ & $95 \mathrm{a}$ \\
\hline Sulfentrazone+diclosulam & $250+25$ & $90 \mathrm{ab}$ & $90 \mathrm{a}$ & $85 \mathrm{a}$ & $95 \mathrm{a}$ & $90 \mathrm{ab}$ & $90 \mathrm{ab}$ & $95 \mathrm{a}$ & $90 \mathrm{ab}$ & $90 \mathrm{a}$ \\
\hline Sulfentrazone+ diclosulam & $500+50$ & $100 \mathrm{a}$ & $95 \mathrm{a}$ & $90 \mathrm{a}$ & $100 \mathrm{a}$ & $95 \mathrm{a}$ & $90 \mathrm{ab}$ & $100 \mathrm{a}$ & 95 a & $90 \mathrm{a}$ \\
\hline Diclosulam & 35 & $85 \mathrm{~b}$ & $85 \mathrm{a}$ & $85 \mathrm{a}$ & $95 \mathrm{a}$ & $85 \mathrm{~b}$ & $85 \mathrm{~b}$ & $95 \mathrm{a}$ & $85 \mathrm{~b}$ & $85 \mathrm{a}$ \\
\hline Diclosulam & 70 & $90 \mathrm{ab}$ & $90 \mathrm{a}$ & $90 \mathrm{a}$ & $100 \mathrm{a}$ & $90 \mathrm{ab}$ & $90 \mathrm{ab}$ & $100 \mathrm{a}$ & $95 \mathrm{a}$ & $90 \mathrm{a}$ \\
\hline C.V. $(\%)$ & - & 7,0 & 3,8 & 4,1 & 5,0 & 6,2 & 5,2 & 4,1 & 5,9 & 3,5 \\
\hline
\end{tabular}


Francisco de Assis Rolim Pereira et al.

Tabela 9 - Efeitos residuais sobre culturas em sucessão aos 30, 45 e 60 dias após a semeadura. Ensaio 1, Solo arenoso. Campo Grande, MS. 1999/2000.

\begin{tabular}{|c|c|c|c|c|c|c|c|c|c|c|c|c|c|c|c|c|c|c|c|}
\hline \multirow{3}{*}{ PRODUTOS } & \multirow{3}{*}{$\begin{array}{l}\text { DOSE } \\
(\mathrm{g} / \mathrm{ha})\end{array}$} & \multicolumn{18}{|c|}{ FITOTOXICIDADE (\%) / CULTURA } \\
\hline & & \multicolumn{3}{|c|}{ Milheto } & \multicolumn{3}{|c|}{ Aveia } & \multicolumn{3}{|c|}{ Sorgo } & \multicolumn{3}{|c|}{ Milho } & \multicolumn{3}{|c|}{ Feijão } & \multicolumn{3}{|c|}{ Algodão } \\
\hline & & 30 & 45 & 60 & 30 & 45 & 60 & 30 & 45 & 60 & 30 & 45 & 60 & 30 & 45 & 60 & 30 & 45 & 60 \\
\hline Testemunha & -- & 0 & 0 & 0 & 0 & 0 & 0 & 0 & 0 & 0 & 0 & 0 & 0 & 0 & 0 & 0 & 0 & 0 & 0 \\
\hline Sulfentrazone & 5000 & 0 & 0 & 0 & 0 & 0 & 0 & 0 & 0 & 0 & 0 & 0 & 0 & 0 & 0 & 0 & 0 & 0 & 0 \\
\hline Sulfentrazone & 1000 & 40 & 30 & 20 & 35 & 30 & 20 & 25 & 15 & 10 & 0 & 0 & 0 & 0 & 0 & 0 & 10 & 10 & 5 \\
\hline Sulfentrazone+diclosulam & $250+25$ & 0 & 0 & 0 & 0 & 0 & 0 & 0 & 0 & 0 & 0 & 0 & 0 & 0 & 0 & 0 & 0 & 0 & 0 \\
\hline Sulfentrazone+diclosulam & $500+50$ & 10 & 5 & 5 & 5 & 5 & 5 & 5 & 5 & 5 & 0 & 0 & 0 & 0 & 0 & 0 & 0 & 0 & 0 \\
\hline Diclosulam & 35 & 0 & 0 & 0 & 0 & 0 & 0 & 0 & 0 & 0 & 0 & 0 & 0 & 0 & 0 & 0 & 0 & 0 & 0 \\
\hline Diclosulam & 70 & 30 & 20 & 15 & 25 & 20 & 15 & 20 & 15 & 10 & 0 & 0 & 0 & 0 & 0 & 0 & 0 & 0 & 0 \\
\hline
\end{tabular}

\section{CONCLUSÕES}

Os herbicidas sulfentrazone e diclosulam, nas doses de $1000 \mathrm{~g} /$ ha e $70 \mathrm{~g} / \mathrm{ha}$, não devem ser aplicados em solo de textura arenosa sobre as cultivares de soja Conquista, Paiaguás e Taquari.

Em solo de textura argilo-arenoso, os tratamentos químicos avaliados não apresentam restrições de seletividade aos cultivares de soja testados (Bacuri, Pioneira, Uirapuru, Taquari, Curió, Campo Grande, Xingu, Tucano, Parecis, Cristalina RC, Garça Branca, CAC-1, Piapara, Canário, Conquista, Paiaguás, Surubi, Pintado, Suprema, FT-2000 e FTEstrela) e não causam efeitos residuais sobre o desenvolvimento das culturas sucessivas à soja (milheto, aveia-preta, sorgo cv. Z-822, milho cv. 8202, feijão cv. carioca e algodão cv. Ita 90).

As culturas de milheto, aveia-preta e sorgo, semeadas em sucessão à soja, apresentam suscetibilidade aos efeitos residuais de sulfentrazone e diclosulam quando aplicados nas doses de $1000 \mathrm{~g} / \mathrm{ha}$ e $70 \mathrm{~g} / \mathrm{ha}$ em pré-emergência na cultura da soja, em solo arenoso.

Os herbicidas sulfentrazone (500 e $1000 \mathrm{~g} / \mathrm{ha})$ diclosulam (35 e $70 \mathrm{~g} / \mathrm{ha}$ ) e as misturas em tanque de sulfentrazone+diclosulam $(250+25$ e $500+50 \mathrm{~g} / \mathrm{ha})$, apresentam eficiência no controle das plantas daninhas Digitaria horizontalis, Amaranthus viridis, Ipomoea grandifolia, Portulaca oleraceae e Bidens pilosa.

\section{LITERATURA CITADA}

DAYAN, F.E.; WEETE, J.D.; DUKE, S.O.; HANCOCK, H.G. Soybean (Glycine max) cultivar diferences in response to sulfentrazone. Weed Science, v. 45, p. 634641, 1997.

DUKE, S.O.; DAYAN, F. E. ; YAMAMOTO, M.; DUKE, M.V.;

REDDY, K.N. Protoporphyrinigen oxidas e inhibtors - their current and future role. Proc. Int. Weed Control Congr., v. 3, p. 775-780, 1996.

EMBRAPA Agropecuária Oeste. Soja: recomendações técnicas para Mato Grosso do Sul e Mato Grosso. Dourados, 1999. 158p. (Embrapa Agropecuária Oeste. Circular Técnica,2).

RODRIGUES, B. N; ALMEIDA, F. S. Guia de Herbicidas, $4^{\mathrm{a}}$ ed. Londrina, 1998. 647p.

WALKER, R. H.; RICHBURG e JONES, R. E. F6285 effi cacy as affcted by rate and method of application. Proc. Weed Sci. Soc. v.45, p.51, 1992. 